\title{
Optimal per-Node Rate Allocation to provide per-Flow End-to-End Delay Guarantees in a Network of Routers supporting Guaranteed Service Class
}

\author{
Aniruddha Diwan \\ ECE Department \\ Indian Institute of Science \\ Bangalore 560012
}

\author{
Joy Kuri \\ CEDT Department \\ Indian Institute of Science \\ Bangalore 560012
}

\author{
Anurag Kumar \\ ECE Department \\ Indian Institute of Science \\ Bangalore 560012
}

\begin{abstract}
In this paper, we investigate the problem of providing worst-case end-to-end delay guarantee to a token bucket constrained flow traversing a series of $N$ packet schedulers. We consider a network of routers that support the Guaranteed Service class of the IETF Integrated Services (IntServ) Working Group; this service class is proposed to provide Quality of Service $(\mathrm{QoS})$ in the Internet. Under this framework, a worst-case end-to-end delay bound to a flow is provided by allocating a rate at each network element on the path of the flow. We associate a cost with allocating a rate at each link. The cost is assumed to be a convex and non-decreasing function of rate. We investigate the problem of obtaining an optimal rate allocation for a flow that minimizes the total cost subject to the delay requirement and the available link capacity constraints. Allocating an identical rate at each link on the path of a flow is a widely used approach under the Guaranteed Service framework. We investigate the optimality of this approach and show that under certain conditions, it need not be optimal. Moreover, we investigate the optimal solution to the total cost minimization problem and give scenarios in which we can explicitly obtain the optimal solution. Based on these results, we present an algorithm for optimal rate allocation that is based on multiple rates. However, with blocking probability as the performance criterion, we find through simulations, that the optimal rate allocation algorithm is only marginally better for connections with longer path lengths at the expense of those with shorter path lengths. We also observe that the performances of both the algorithms are very close and so, in practice, the simpler identical rate algorithm may be sufficient.
\end{abstract}

\section{INTRODUCTION}

In this paper we consider the problem of providing Quality of Service (QoS) guarantees to the applications of the Internet. Recent technological advances have given rise to a plethora of diverse multimedia applications and the Internet is expected to support such applications having various QoS requirements. Service guarantees are required in terms of not only channel reliability but also throughput and end-to-end packet delays. In order to meet the QoS requirements of connections, the Internet needs to support various network mechanisms. A QoS routing mechanism is required which selects a suitable unicast path (or a multicast tree) for the packets of a connection. Once a path is selected, a mechanism is needed that provides QoS guarantees by reserving resources such as bandwidth/buffer on the network elements (NE) along the path of the connection. As there is a local cost associated with allocation of resources at a network element, the resources should be optimally allocated to minimize the overall cost. This can be formulated as a

This work was carried out at the Indian Institute of Science and supported by a research grant from HFCL India. network optimization problem for mapping of end-to-end QoS requirements to local requirements along a path. Addressing this problem is important because it impacts the routing process as well as the reservation of resources along the path.

In our work, the QoS requirements of a connection are specified in terms of throughput and end-to-end delay and these QoS requirements are guaranteed by allocating a rate on each link along the path of a connection. In particular, we consider the Guaranteed Service [1] framework of the Integrated Services (IntServ) charter [2] of the IETF, defined to address the problem of supporting applications with QoS requirements in the Internet. It requires the traffic of a data flow to conform to certain parameters (specified by the data flow at the time of connection setup) and provides an assured level of bandwidth, a firm end-to-end delay bound and no queueing loss for conforming packets of the data flow. The delay bound is determined by the rates allocated on the links of the path. One needs to allocate appropriate rates on the links of the path so that the delay bound does not exceed the end-to-end delay requirement. We associate a cost with allocation of a rate on each link. In this paper, we investigate the problem of optimal mapping of the throughput and end-to-end delay requirements to local rates; the optimality criterion is minimization of the total cost of rate allocation.

Many papers have reported work on QoS mapping problems in which the end-to-end QoS requirements are mapped into local requirements. Since the problem investigated in this paper falls under this category, it is important to compare and contrast our work against the related work. [3], [4], [5], [6], [7] report work on QoS partitioning where the problem is to optimally partition an end-to-end QoS requirement into local QoS requirements. However, these works consider the class of additive QoS parameters such as delay and jitter. Thus the problem is to obtain an optimal local delay partition for an endto-end delay requirement that minimizes the cost. Though the general problem of partitioning additive QoS parameters is intractable, these works restrict themselves to particular forms of cost functions and obtain efficient solutions. The QoS mapping problem that we consider does not fit in the frameworks above because in our case, the end-to-end delay bound is not determined by the local delay bounds, but by the rates allocated on the links of the path of a connection.

The problem of providing QoS guarantees under the Guaranteed Services framework has been studied in [8], [9]. Though the issues addressed in these works are different, the approach 
for providing end-to-end delay guarantees remains the same. The approach is as follows. Consider a data flow traversing a series of $N$ links. An identical rate is allocated on all the links on the path of the flow. The minimum rate $R$ required to guarantee this delay can be computed from the delay bound expression. If rate $R$ is available on the links of the route of the flow, the flow is admitted and otherwise it is rejected. This single rate approach has the following drawbacks. Firstly, it need not minimize the total cost of rate allocation. Secondly, it does not take into consideration the extra available resources. That is, if the computed rate $R$ is not available on only a few links, it might still be possible to admit the connection by giving higher rates on the non-bottleneck links. We address this issue by permitting allocation of different rates instead of an identical rate. Note that. in [10] the author has implicitly recognized the limitations of the single rate approach and deals with this issue through the use of "slack terms". However, probably since the focus of the paper was RSVP, the issue was not addressed adequately in it. Also it does not address the important problem of computing rates that minimize the total cost.

\section{A. Our Contributions}

- In this paper, we explore the end-to-end delay bound to optimally allocate rates for connections traversing a series of routers that support Guaranteed Service class. We investigate whether or not the widely used identical rate allocation approach is optimal (i.e., minimizes the total cost). It turns out that under certain conditions related to the router parameters and flow parameters, this need not be true.

- We investigate the optimal solution to the total cost minimization problem and give scenarios in which we can explicitly obtain the structure of the optimal solution. Note that the problem of minimizing the total allocated rate is one special case in which the cost is the rate itself. So our results are directly applicable here.

- For the case when the router parameters along the path are the same, we present an optimal rate allocation scheme based on the theoretical results and compare its simulated performance against that of the identical rate allocation scheme. We try to judge merits and demerits of both approaches based on the simulation results.

\section{The Guaranteed Service Delay Bound}

The Guaranteed Service requires that the flow specify its characteristics in terms of token bucket parameters and the flow traffic is required to conform to these parameters. The token bucket parameters are a triplet $(b, r, p)$, where $b$ is the token bucket size, $r$ is the token accumulation rate, and $p$ is the maximum peak rate of the flow. The maximum packet size, denoted by $L$, is also required. Network element (i.e., router interface) $i$ exports parameters $C_{i}$ and $D_{i}$, that quantify the level of service that it can provide to flows that traverse it. These exported parameters are interpreted in the context of a rate $R_{i}$ that might be reserved for a flow. Typically, $C_{i}$ and $D_{i}$ approximate the departure from the "fluid model" of service. Consider a flow traversing a series of $N$ network elements and let $R_{i}$ be the rate allocated at NE $i$ on the path of the flow. Let $R_{\text {min }}=\min _{i} R_{i}$. With the flow parameters $(b, r, p, L)$ and the parameters $\left(C_{i}, D_{i}\right)$ of the routers on the path known, the endto-end delay bound for the packets of the flow is as follows ${ }^{1}$ [8], [12], [13].

$$
D^{\text {bound }}=\left\{\begin{array}{l}
\frac{(b-L)\left(p-R_{\min }\right)}{R_{\min }(p-r)}+ \\
\frac{L}{R_{\min }}+\sum_{i=1}^{N}\left[\frac{C_{i}}{R_{i}}+D_{i}\right], \quad p>R_{\min } \\
\frac{L}{R_{\min }}+\sum_{i=1}^{N}\left[\frac{C_{i}}{R_{i}}+D_{i}\right], \quad p \leq R_{\min }
\end{array}\right.
$$

\section{PROBLEM Formulation}

Consider a flow that is to be admitted on a path consisting of $N$ links. Let $(b, r, p)$ be the token bucket parameters and $L$ be the maximum packet size of the flow. Link $i$ exports parameters $C_{i}$ and $D_{i}$. If $R_{i}$ is the rate allocated to the flow on link $i$, $D^{\text {bound }}$, computed from eqn. (1) is the maximum end-to-end delay encountered by the packets of the flow. We call $\bar{R}=$ $\left\{R_{1}, \cdots, R_{N}\right\}$ as a rate vector. Let $D^{\text {reqd }}$ be the maximum end-to-end delay that is acceptable for the packets of the flow. Then the flow can be admitted if we can obtain a rate vector $\bar{R}$ such that,

1) $D^{\text {bound }} \leq D^{\text {reqd }}$.

2) $R_{i} \leq \gamma_{i}, 1 \leq i \leq N$, i.e., the allocated rate $R_{i}$ is less than the available link bandwidth $\gamma_{i}$.

3) $R_{\min }=\min _{i} R_{i} \geq r$, i.e., the minimum allocated rate is at least the average arrival rate of the flow.

There may be many rate vector assignments that satisfy the above constraints. We associate a cost with allocating a rate on a link. The cost function $f_{i}\left(R_{i}\right)$ for link $i$ is assumed to be convex and non-decreasing in the allocated rate $R_{i}$ on link $i$ for a flow. It is further assumed that the cost function is the same for each link and is denoted by $f()$. For a rate vector $\bar{R}=\left\{R_{1}, \cdots, R_{N}\right\}$, the total cost is defined as $t(\bar{R})=\sum_{i=1}^{N} f\left(R_{i}\right)$. We would like to choose that rate vector which minimizes the total cost and hence our optimization criterion is minimization of the total cost for the flow.

In the rest of the paper, we investigate the problem of obtaining a rate vector for which $t(\bar{R})$ is minimized. Also we consider only rate vector assignments for which $p>R_{\min }$ though the work is applicable to the case $p<R_{\min }$ as well. Thus, we always work with the first part of the delay bound of eqn. (1) for simplicity. Without loss of generality, we number the $N$ links of the path as follows. The link with the least available capacity is numbered 1 and so on, i.e., $\gamma_{1} \leq \gamma_{2} \leq \cdots \leq \gamma_{N}$.

\footnotetext{
${ }^{1}$ Note that $R_{\min } \geq r$ and when $R_{\min }<r$ the delay is unbounded.
} 
We now present the minimization problem which we denote by MinimumCost.

(MinimumCost) $\min \sum_{i=1}^{N} f\left(R_{i}\right)$

subject to:

$$
\begin{aligned}
& \left.\frac{(b-L)\left(p-R_{\min }\right)}{R_{\min }(p-r)}+\frac{L}{R_{\min }}+\sum_{i=1}^{N}\left[\frac{C_{i}}{R_{i}}+D_{i}\right] \leq D^{\mathrm{reg} 2}\right) \\
& R_{i} \leq \gamma_{i}, 1 \leq i \leq N \\
& R_{\min } \geq r
\end{aligned}
$$

Note that if $R_{1}=R_{2}=\cdots=R_{N}=r$ is a feasible solution to the MinimumCost problem, it is also the optimal solution. This is because we can not reduce $R_{i}$ further. To avoid this trivial case, in the rest of the paper, we assume that $(r, r, \cdots, r)$ is not a feasible solution to the MinimumCost problem.

Let $\sigma=\frac{(b p-r L)}{(p-r)}, D=D^{\text {reqd }}-\sum_{i=1}^{N} D_{i}-\frac{(b-L)}{(p-r)}$. Let $x_{i}=$ $1 / R_{i}, 1 \leq i \leq N, \theta_{i}=1 / \gamma_{i}, 1 \leq i \leq N$ and $\delta=1 / r$. Let $\bar{x}=\left(x_{1}, \cdots, x_{N}\right)$ and $x_{\max }=\max _{i} x_{i}$. Let $h\left(x_{i}\right)=f\left(1 / x_{i}\right)$. We give a fact about $h\left(x_{i}\right)$.

Lemma III.1: If $f(x)$ is a convex non-increasing (convex non-decreasing) function of $x$, then $h(x)=f(1 / x)$ is a convex non-decreasing (convex non-increasing) function of $x$.

In this paper we omit proofs of the results due to lack of space, please refer to [11] for the proofs. We now present the MinimumCost problem in terms of $h(), x_{i}, \theta_{i}$ and $\delta$.

$$
\text { (MinimumCost) } \min \sum_{i=1}^{N} h\left(x_{i}\right)
$$

subject to:

$$
\begin{aligned}
& \sigma x_{\max }+\sum_{j=1}^{N} C_{j} x_{j} \leq D \\
& x_{i} \geq \theta_{i}, \quad 1 \leq i \leq N \\
& x_{i} \leq \delta, \quad 1 \leq i \leq N
\end{aligned}
$$

Let $H(\bar{x})=\sum_{i=1}^{N} h\left(x_{i}\right)$ where $\bar{x}=\left(x_{1}, \cdots, x_{N}\right)$.

Lemma III. 2: $H(\bar{x})$ is a convex function in $\bar{x}$.

Note that if the MinimumCost problem is feasible, the flow can be admitted and the optimal solution to the problem gives the optimal rate allocation vector. If the problem is infeasible, the flow is blocked because of lack of sufficient bandwidth.

\section{Problem Solution}

It can be seen that the structure of the MinimumCost problem has a convex cost function with linear constraints. There exist necessary and sufficient conditions in terms of Lagrange multipliers for a feasible solution to be optimal for such optimization problems [14]. Though it seems difficult to explicitly characterize the optimal solution to the MinimumCost problem, we investigate optimality of the identical rate allocation approach in this section. We also explicitly characterize structure of the optimal solution for the case where link parameters $C_{i}$ on the path are equal.

\section{A. The Unbounded Link Capacities Case and Non-optimality of the Identical Rate Vector}

We first investigate the problem when there are no available link capacity constraints, i.e., we can allocate any amount of bandwidth on the links, $\theta_{i}=0,1 \leq i \leq N$. We denote by UnbddRates, the MinimumCost problem without the available link bandwidth constraints, viz.,

(UnbddRates) $\min \sum_{i=1}^{N} h\left(x_{i}\right)$ subject to:

$$
\begin{aligned}
& \sigma x_{\max }+\sum_{j=1}^{N} C_{j} x_{j} \leq D \\
& x_{i}>0, \quad 1 \leq i \leq N \\
& x_{i} \leq \delta, \quad 1 \leq i \leq N
\end{aligned}
$$

Now suppose we decide to allocate identical rate to the flow on each link of its route. It is easy to compute the minimum identical rate $R_{\text {equal }}$ from the delay bound constraint of eqn. (8) which gives,

$$
\begin{aligned}
& x_{\text {equal }}=\frac{D}{\sigma+\sum_{i=1}^{N} C_{i}}, R_{\text {equal }}=\frac{\sigma+\sum_{i=1}^{N} C_{i}}{D} . \\
& \text { Let } \bar{x}_{\text {equal }}=\quad\left(x_{\text {equal }}, \cdots, x_{\text {equal }}\right), \bar{R}_{\text {equal }}= \\
& \left(R_{\text {equal }}, \cdots, R_{\text {equal }}\right) .
\end{aligned}
$$

Thus $\bar{x}_{\text {equal }}$ is a feasible solution to the UnbddRates problem.

Note that, allocating $\bar{R}_{\text {equal }}$ is a widely used approach [8], [9] to provide end-to-end delay guarantees under the Guaranteed Services framework. Our next theorem states that $\bar{R}_{\mathrm{equal}}$ need not always be the optimal solution and gives an explicit condition for $\bar{R}_{\text {equal }}$ to be or not to be the optimal solution.

Theorem IV.1: If $\sigma+\sum_{j=1}^{N} C_{j}>N C_{i}, 1 \leq i \leq N, \bar{R}_{\text {equal }}$ is the optimal solution to the UnbddRates problem; otherwise $\bar{R}_{\text {equal }}$ is not the optimal solution to the UnbddRates problem.

We now illustrate the non-optimality of identical rate vector with an example. Let us consider the simple cost function $f\left(R_{i}\right)=R_{i}$ which means $h\left(x_{i}\right)=1 / x_{i}$. Thus our optimization criterion is the minimization of the total bandwidth allocated to the flow. Consider the following choice of parameters.

- $N=3$ and $\sigma=30, C_{1}=25, C_{2}=5, C_{3}=5, r=$ $1, D=6.5$.

Then it can be seen that $\sigma+\sum_{i=1}^{3} C_{i}>3 C_{2}$ and $\sigma+$ $\sum_{i=1}^{3} C_{i}>3 C_{3}$, but $\sigma+\sum_{i=1}^{3} C_{i}<3 C_{1}$.

- The identical rate vector is $\bar{x}_{\text {equal }}=(0.1,0.1,0.1)$ for which the total cost is $H\left(\bar{x}_{\mathrm{e} q u a l}\right)=30$.

- Consider $\bar{x}^{\prime}=\left(x_{1}^{\prime}, x_{2}^{\prime}, x_{3}^{\prime}\right)$ where $x_{1}^{\prime}=0.095, x_{2}^{\prime}=$ $0.103+\frac{0.005}{35}, x_{3}^{\prime}=0.103$. It can be easily verified that $\bar{x}^{\prime}$ is a feasible solution and $H\left(\bar{x}^{\prime}\right)=29.93$. 
This indicates that $\bar{x}_{\text {equal }}$ is not the optimal solution.

Note that, from Theorem IV.1, the identical rate vector is optimal when $\sigma+\sum_{j=1}^{N} C_{j}>N C_{i}, 1 \leq i \leq N$. Consider the case when $C_{1}=C_{2}=\cdots=C_{N}$. It can be seen that $\sigma+\sum_{j=1}^{N} C_{j}>N C_{i}, 1 \leq i \leq N$ and therefore $\bar{x}_{\text {equal }}$ is the optimal solution in this case. We state this as a corollary.

Corollary IV.1: For the case $C_{1}=C_{2}=\cdots=C_{N}, \bar{x}_{\text {equal }}$ is the optimal solution to the UnbddRates problem and therefore $\bar{R}_{\text {equal }}$ is the optimal rate vector for the UnbddRates problem.

Note that $C_{i}$ corresponds to the MTU size at the interface $i$ and it could be different for different links. In the special case of equal MTU, $C_{1}=C_{2}=\cdots=C_{N}$. In the rest of the paper we work with this assumption and use $C$ for $C_{i}$.

Now suppose that the optimal rate $R_{\text {equal }}$ is not available on some links of the route. In most of the approaches [8], [9], the flow is blocked. But, in fact, the delay bound eqn. (1) allows us to allocate different rates at different links on the route of the flow. Thus there might exist another rate vector which satisfies the delay constraint and the available link bandwidth constraints and it might be possible to admit the flow. Therefore, we need to know the optimal rate vector when there are constraints on the available link capacities. We address this problem next.

\section{B. The Bounded Link Capacities Case}

In this section we consider the case when $\bar{R}_{\text {equal }}$ is not available on some of the links. That is, there is at least one link having capacity less than $\bar{R}_{\text {equal }}$. Because of our numbering convention, link 1 is such a link with least capacity. Note that i.e., $\gamma_{1}<R_{\text {equal }}$ and $\theta_{1}>x_{\text {equal }}$.

Lemma IV.1: Let $\bar{x}=\left(x_{1}^{*}, x_{2}^{*}, \cdots, x_{N}^{*}\right)$ be the optimal solution to the bounded link capacities problem. Then $x_{1}^{*}=\theta_{1}$ and $x_{i}^{*} \leq \theta_{1}, 2 \leq i \leq N$.

We first consider the simple case where only link 1 has a capacity $<\bar{R}_{\text {equal }}$. The other links have unbounded link capacities. We formulate the SingleLink problem in terms of $h(), x_{i}, \theta_{1}, \delta$ as follows.

(SingleLink) $\min \sum_{i=1}^{N} h\left(x_{i}\right)$

subject to:

$$
\begin{aligned}
& \sigma x_{\text {max }}+\sum_{j=1}^{N} C x_{j} \leq D \\
& x_{1} \geq \theta_{1}>x_{\text {equal }} \\
& x_{i}>0, \quad 2 \leq i \leq N \\
& x_{i} \leq \delta, \quad 1 \leq i \leq N
\end{aligned}
$$

Using Lemma IV.1, we know that, in the optimal solution of the SingleLink problem, $x_{1}^{*}=\theta_{1}$. If we substitute this in the above SingleLink problem, we have a reduced problem in which
- The delay constraint equation (11) is simplified because $x_{\max }$ can be eliminated.

- We have $(N-1)$ variables.

This is then the UnbddRates problem. It is clear from the solution of the UnbddRates problem that, for the SingleLink case, $x^{*} 2=\cdots=x_{N}^{*}$. Let $R_{\text {equal }}^{1}=\frac{(N-1) C}{D-\frac{\sigma+C}{\gamma_{1}}}$ and let $x_{\text {equal }}^{1}=1 / R_{\text {equal }}^{1}$. With $x_{1}^{*}=\theta_{1}$ known, it can be shown that $x^{*} 2=\cdots=x_{N}^{*}=x_{\text {equal }}^{1}$. Let $\bar{R}_{\text {SingleLink }}=\left(\gamma_{1}, R_{\text {equal }}^{1}, \cdots, R_{\text {equal }}^{1}\right)$ and $\bar{x}_{\text {SingleLink }}=$ $\left(\theta_{1}, x_{\text {equal }}^{1}, \cdots, x_{\text {equal }}^{1}\right)$.

Lemma IV.2: $\bar{x}_{\text {Single Link }}$ is the optimal solution to the SingleLink problem and therefore $\bar{R}_{\text {SingleLink }}$ the optimal rate allocation vector for the SingleLink problem.

Now it is possible that $R_{\mathrm{e} q u a l}^{1}$ is not available on link 2. This leads to the 2-Links problem, in which the bandwidth available on link 1 is $\gamma_{1}<R_{\text {equal }}$ and the bandwidth available on link 2 is $\gamma_{2}<R_{\text {equal }}^{1}$ and unlimited bandwidth is available on the links $3, \cdots, N$. From lemma IV.1, we know that $x_{1}^{*}=\theta_{1}$ for the 2-Links case. By using arguments similar to the SingleLink case, we can prove that $x_{2}^{*}=\theta_{2}$ and $x_{3}^{*}=\cdots=x_{N}^{*}$. Thus, by applying the SingleLink case arguments in an iterative manner, we can prove the general case of $K$ links with bounded capacities. We state this now. Let $R_{\text {equal }}^{I}=\frac{(N-I) C}{D-\frac{\sigma+C}{\gamma_{1}}-\frac{C}{\gamma_{2}}-\cdots-\frac{C}{\gamma_{I}}}, I \geq 1$ and $x_{\text {equal }}^{I}=$ $1 / R_{\text {equal }}^{I}$. Let $\bar{R}_{I \mathrm{Links}}=\left(\gamma_{1}, \cdots, \gamma_{I}, R_{\text {equal }}^{I}, \cdots, R_{\text {equal }}^{I}\right)$ and $\bar{x}_{\text {ILinks }}=\left(\theta_{1}, \cdots, \theta_{I}, x_{\text {equal }}^{I}, \cdots, x_{\text {equal }}^{I}\right), I \geq 1$. The $K$-Links structure is as follows.

- The available link capacity on link $I, 1 \leq I \leq K$ is $\gamma_{I}$, where $\gamma_{I}<R_{\text {equal }}^{(I-1)}$ and unlimited capacity is available on links $(K+1), \cdots, N$.

It can be proved using the principle of induction that,

Theorem IV.2: $\bar{x}_{K \text { Links }}$ is the optimal solution to the KLinks problem and therefore $\bar{R}_{K \text { Links }}$ is the optimal rate allocation vector for the K-Links problem.

Note that in practice, there is an available link capacity constraint on every link. In the next section, we present an algorithm which tells us whether or not we can admit a flow and in case we can admit a flow, it also gives the optimal rate allocation vector.

\section{Optimal Rate Allocation Vector Algorithm}

Based on the results obtained in the previous sections, we develop an algorithm for optimal rate allocation for a flow. In iteration $i$, the algorithm maps the rate allocation problem into some $K_{i}$ Links problem and checks for availability of the computed rate $R_{\text {equal }}^{\left(K_{i}\right)}$ on links $K_{i}+1, \cdots, N$. If the rate is available on all the links, the flow is admitted, otherwise the algorithm computes $K_{i+1}$ and moves to next iteration (i.e., $i+1$ ). Note that, $K_{i+1}>K_{i}$. When $K_{i+1}=N$, it is clear that sufficient 
bandwidth is not available on any link and therefore the flow has to be blocked. The optimal rate allocation algorithm is as follows,

1) Compute the identical rate $R_{\text {equal }}$.

2) If $R_{\text {equal }}$ is available on every link, we can accept the flow and $\bar{R}_{\text {equal }}$ is the optimal rate allocation vector. Exit.

3) If $R_{\text {equal }}$ is not available on each the of the links $1, \cdots, N$, the flow can not be admitted. Block the flow. Exit.

4) The required rates are not available on the first $I$ links. Compute $R_{\mathrm{e} q u a l}^{I}=\frac{(N-I) C}{D-\frac{\sigma+C}{\gamma_{1}}-\frac{C}{\gamma_{2}}-\cdots-\frac{C}{\gamma_{I}}}$.

5) If $R_{\text {equal }}^{I}$ is available on links $I+1, \cdots, N$, then the flow can be admitted and $R_{1}=\gamma_{1}, R_{2}=\gamma_{2}, \cdots, R_{I}=$ $\gamma_{I}, R_{(I+1)}=\cdots=R_{N}=R_{\text {equal }}^{I}$ is the optimal rate allocation vector. Exit.

6) If $R_{\text {equal }}^{I}$ is not available on each of the links $I+$ $1, \cdots, N$, the flow can not be admitted. Block the flow. Exit.

7) If $R_{\mathrm{e} q u a l}^{I}$ is not available on links $I+1, \cdots, J<N$, then set $I=J$. Go to Step 4 .

\section{Simulation Results}

In the previous sections, we have seen that the optimal rate algorithm might admit a connection when the identical rate allocation algorithm blocks it. In this section, we explore the performance of these algorithms in a dynamic setting where connection requests arrive and depart randomly. In particular, we investigate whether or not the optimal rate algorithm performs better than the identical rate algorithm. Therefore, in this section, we compare the simulated performance of the optimal rate allocation algorithm with that of the identical rate allocation algorithm.

The network under consideration is shown in Fig. 1. iAt each node, the packets are scheduled according to the Weighted Fair Queueing (WFQ) policy [15], [16]. WFQ falls under the Guaranteed Services framework with the following parameters. For a flow $i$ at link $l, C_{l}=L_{i}^{\max }$ and $D_{l}=\frac{L^{\mathrm{max}}}{g_{l}}+d_{l}^{\text {prop }}$, where $L_{i}^{\max }$ is the maximum packet size of flow $i, L^{\max }$ is the maximum size of the packets at link $l, g_{l}$ is the capacity of link $l$ and $d_{l}^{\text {prop }}$ is the propagation delay of link $l$.

In our simulations, we assume that all the connections to be routed are full-duplex, that all links are bidirectional and the two halves of a full-duplex connection are to be routed on the same path. We consider the shortest path (in the number of hops) for a source-destination pair. The paths can be given or can be computed given the network topology. Connection requests are assumed to arrive according to a Poisson process and last for a duration that is exponentially distributed with unit mean. We further assume that an arriving connection request belongs to one of the pre-specified traffic classes of table I [8].
Each traffic class is identified by its token bucket parameters, maximum packet size and end-to-end delay requirement. We restrict ourselves to the uniform traffic case, i.e., load of each class on each source destination pair is uniformly distributed. The performance criterion is the blocking probability of each class. With this setup, we obtain blocking probability curves.

We first show that the optimal rate allocation algorithm performs marginally better than the identical rate allocation algorithm, for connections that traverse longer paths. For this we assume that only video conferencing connections arrive and obtain separate blocking probability curves for connections with 1-hop, 2-hops and 3-hops. It can be seen from the plot of Fig. 2 that, with the optimal rate algorithm, there is a reduction in the blocking probability for connections with 3-hops at the expense of an increase in the blocking probability for connections with 1-hop and 2-hops.

Next we consider the case when arriving connections belong to the traffic classes of table I. From Fig. 3 and Fig. 4, it can be seen that, the blocking probability curves are almost the same for both rate allocation algorithms. So, despite the fact that a connection which is blocked by the identical rate algorithm, might be admitted by the optimal rate algorithm, the performance of both the algorithms is almost the same. This is probably because the optimal rate allocation algorithm admits connections at the cost of higher network bandwidth. This leaves less bandwidth for future calls and leads to almost the same performance. Thus, we can conclude that, the simpler identical rate allocation algorithm can be sufficient for connection admission.

\section{CONCLUSIONS}

In this work, we have investigated the problem of optimal rate vector allocation to provide end-to-end delay guarantees to Guaranteed Service class connections. We have shown that the widely used identical rate allocation algorithm need not be optimal (in minimizing the overall cost of rate allocation) when router parameters and flow specifications do not satisfy certain conditions. We have also shown that we can explicitly obtain the optimal solution to minimize the total cost when the parameters at each router are identical. We have presented an optimal rate vector computation algorithm for this case. We have found through simulations that the optimal rate allocation algorithm only marginally reduces blocking probabilities, for connections traversing longer paths. Moreover, the performance of identical rate allocation algorithm is very close to that of the optimal rate allocation algorithm. Therefore, owing to the simplicity of the identical rate allocation algorithm, we can use it for connection admission in practice. Certain issues need to be investigated further. One such issue is computation of the optimal rate vector when router parameters are not identical. Also the more general total cost minimization problem in which link cost functions are not the same, requires further investigation. The current RSVP structure supports only the 
identical rate reservation and RSVP based scheme for supporting reservation of a rate vector are required. These issues form part of our ongoing work.

\section{REFERENCES}

[1] S. Shenker and C. Partridge and R. Guérin, "Specification of guaranteed quality of service", Request For Comments (Proposed Standard) RFC 2212, IETF, Sept. 1997.

[2] Integrated Services Charter, http://www.ietf.org/html.charters/intservcharter.html.

[3] R. Nagarajan and J. Kurose and D. Towsley, "Allocation of Local Quality of Service Constraints to meet End-to-End Requirements", In IFIP Workshop on the Performance Analysis of ATM Systems, Martinique, Jan. 1993.

[4] D. Lorenz and A. Orda, "Optimal Partition of QoS Requirements on Unicast Paths and Multicast Trees", Proceedings of the IEEE INFOCOM, 1999.

[5] R. Guérin and A. Orda, "QoS-based Routing in Networks with Inaccurate Information: Theory and Algorithms", IEEE/ACM Transactions on Networking, Vol. 7, No. 3, pp. 350-364, Jun. 1999.

[6] D. Lorenz and A. Orda, "QoS Routing on Networks with Uncertain Parameters", IEEE/ACM Transactions on Networking, Vol. 6, No. 6, pp. $768-778$, Dec. 1998

[7] D. Raz and Y. Shavitt, "Optimal Partition of QoS Requirements with Discrete Cost Functions", Proceeding of the IEEE INFOCOM, 2000.

[8] L. Georgiadis, R. Guérin, V. Peris and R. Rajan, "Efficient support of delay and rate guarantees in an Internet", Proc. of SIGCOMM, pp. 106116, August 1996

[9] R. Guérin and V. Peris, "Quality-of-Service in packet networks: basic mechanisms and directions", Computer Networks, vol. 31, no. 3, pp. 169179, Feb. 1999.

[10] P. White, "RSVP and integrated services in the Internet", IEEE Coтmunications Magazine, May 1997.

[11] A. Diwan, J. Kuri and A. Kumar, "Optimal per-Node Rate Allocation to provide per-Flow End-to-End Delay Guarantees in a Network of Routers supporting Guaranteed Service Class", Technical Report, ECE Dept., IISc, Aug. 2001. http://www.ece.iisc.ernet.in/ diwan/papers/optimalrate.ps

[12] R. Agrawal and R. Rajan, "Performance bound for guaranteed and adaptive services", IBM Research Report, RC20649, Dec. 1996.

[13] D. Stiliadis and A. Varma, "Latency-rate servers: a general model for analysis of traffic scheduling algorithms", IEEE/ACM Transactions on Networking, Oct. 1998.

[14] D. Bertsekas, Nonlinear Programming, Athena Scientific, Belmound, Massachusetts, 1995.

[15] A. Demers, S. Keshav and S. Shenker, "Analysis and simulation of a fair queueing algorithm", Proc. ACM SIGCOMM'89, pp. 3-12, 1989.

[16] A. K. Parekh and R. G. Gallagher,"A generalized processor sharing approach to flow control in integrated services networks: the single node case", IEEE Trans. on Networking, vol. 1, no. 3, pp. 137-150, 1993.

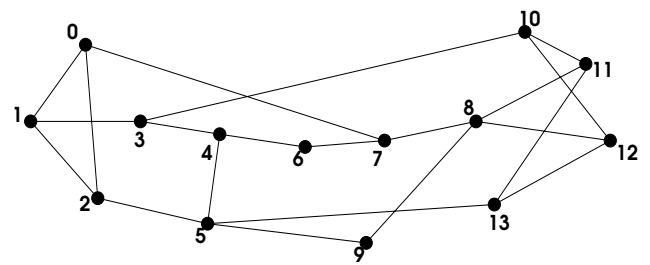

Fig. 1. The network under consideration. Each link has maximum capacity 155 Mbps and propagation delay $4 \mathrm{~ms}$.
TABLE I

PARAMETERS FOR TRAFFIC MODELS OF TYPE VOICE AND VIDEO.

\begin{tabular}{|l|c|c|c|c|c|}
\hline Class & $\begin{array}{c}b \\
(\mathrm{kB})\end{array}$ & $\begin{array}{c}r \\
(\mathrm{Mbps})\end{array}$ & $\begin{array}{c}p \\
(\mathrm{Mbps})\end{array}$ & $\begin{array}{c}D^{\text {reqd }} \\
(\mathrm{ms})\end{array}$ & $\begin{array}{c}L \\
(\mathrm{kB})\end{array}$ \\
\hline voice & 0.1 & 0.064 & 0.064 & 50 & 0.1 \\
\hline video conf & 10 & 0.5 & 10 & 75 & 1.5 \\
\hline st video & 100 & 3 & 10 & 100 & 1.5 \\
\hline
\end{tabular}

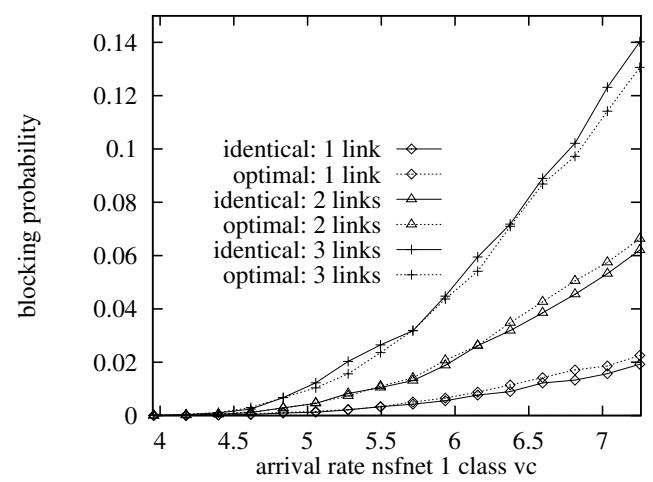

Fig. 2. Blocking probabilities of identical rate allocation and optimal rate allocation for connections with various hop counts are plotted. Only video conferencing connections are present. Note the marginal reduction in blocking probability for 3 -hop connections.

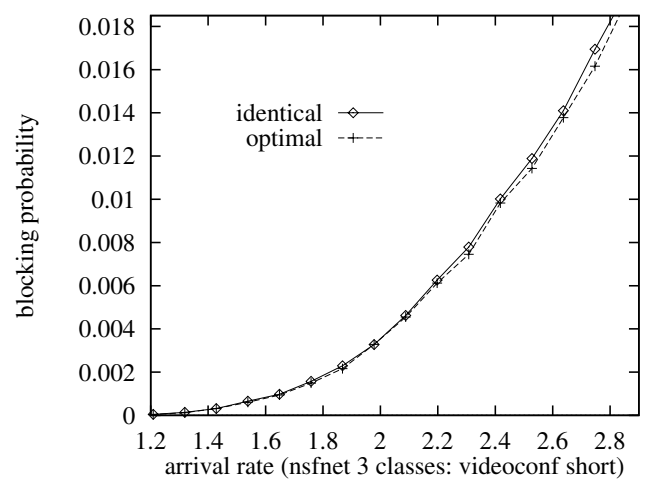

Fig. 3. Blocking probabilities of video conferencing calls for identical rate allocation and optimal rate allocation are plotted. The blocking probabilities are almost the same.

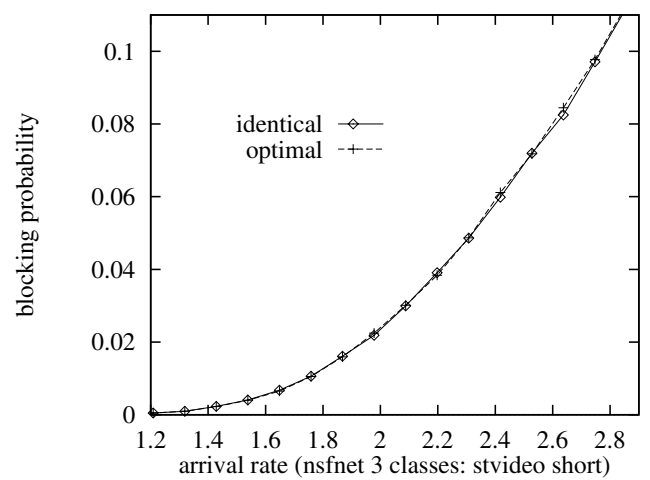

Fig. 4. Blocking probabilities of stored video calls for identical rate allocation and optimal rate allocation are plotted. The blocking probabilities are almost the same. 